\title{
Angiopoietin-2 and vascular complications of type 2 diabetes
}

\section{ABSTRACT}

Cardiovascular diseases are the leading cause of death among patients with diabetes mellitus type 2 (T2DM), and microvascular complications of diabetes are associated with high morbidity and diminished quality of life. Angiopoietin-2 (Ang-2) is essential for endothelial physiology and plays an important role in vascular-related diseases. Its concentration in blood is elevated in patients with T2DM in comparison to those with normal glucose tolerance and in subjects with diabetic macrovascular complications compared to those without them. As for microvascular complications, it was found that serum Ang-2 concentration was significantly higher among T2DM patients with diabetic retinopathy compared to diabetic patients free of this complication. Moreover, in an animal model, Ang-2 mRNA expression was elevated in endothelial cells isolated from diabetic mice's kidneys when compared to non-diabetic controls, which suggests its role in the development and progression of diabetic nephropathy. Targeting molecular Ang-2 pathway may become a therapeutic aim, especially that anti-angiogenic therapies are considered to be effective treatment methods in this field. (Clin Diabetol 2020; 9; 3: 201-204)

Key words: diabetes mellitus, biomarker, vascular complications, angiopoietin-2, angiopoietin

Address for correspondence:

lek. Tomasz Skowerski

Katedra i Klinika Kardiologii

Wydział Nauk o Zdrowiu

Śląski Uniwersytet Medyczny

ul. Ziołowa 45, 40-635 Katowice

Phone: +48-607-234-440

e-mail: tskowerski@gmail.com

Clinical Diabetology 2020, 9, 3, 201-204

DOI: $10.5603 /$ DK.2020.0009

Received: 14.11 .2019

Accepted: 01.01.2020

\section{Introduction}

451 million people globally have diabetes, with the vast majority of them suffering from T2DM, and its prevalence is projected to reach 693 million people by the year 2045 [1]. Cardiovascular diseases (CVD) are the leading cause of death among patients with T2DM and diabetes reduces life expectancy curve by as much as ten years [2,3]. The microvascular complications of diabetes are responsible for increased morbidity and diminished quality of life. Diabetic retinopathy became a leading cause of vision loss, diabetic nephropathy is a primary cause of end-stage renal disease, and the diabetic foot is the most often non-traumatic amputations of lower extremities [4, 5].

Endothelial dysfunction is an important factor involved in the pathogenesis of diabetes-related macroand microangiopathies [6]. Angiogenesis and vascular remodeling are modulated by vascular growth factors, among others, angiopoietins with two major ones well characterized, which are angiopoietin-1 (Ang-1) and Ang-2 [7, 8].

Ang-2 is exclusively expressed in the endothelium cells, and both Ang-1 and Ang-2 bind to the receptor Tie-2 (tyrosine kinase with immunoglobulin and epidermal growth factor homology domains-2) with the same affinity $[9,10]$. Ang-2 possesses the opposite physiological properties to Ang-1. Ang-1 signaling through Tie-2 is responsible for endothelial wall stabilization and protects the endothelium from excessive activation by growth factors and cytokines [11], whereas the rapid release of Ang-2 from epithelium can prevent Ang-1 from binding to the receptor and in this manner disrupt the protective Ang-1/Tie-2 signaling [8, 11, 12].

Expression of Ang-2 is influenced by inflammatory factors like thrombin [13] and conditions like hypoxia [13]. There is some evidence for the association of elevated concentration of circulating Ang- 2 with the incidence 
of CVD [14] and cardiovascular mortality in the general population [15]. Dysfunction in angiogenesis has also been proposed as a common factor predisposing to vascular complications in diabetes [16].

Moreover, the selective increase in plasma Ang-2 concentration may lead to neovascularization and endothelial abnormalities, which are linked to the pathophysiology of microvascular and atherosclerotic complications in T2DM [17]. In the study by Lim et al. it was proven for the first time that plasma Ang-2 concentration is elevated among patients with diabetes in comparison to the ones without glucose metabolism disorders [18].

The aim of this review article is to present scientific data related to Ang- 2 and its association with microand macro- vascular complications in T2DM.

\section{Ang-2 and macroangiopathy}

Rasul et al. reported that subjects with diabetic macrovascular complications, in particular with the CVD, had higher serum Ang-2 concentration than those without these complications [19]. This was in line with previous in vitro study, proving that hyperglycemia may lead to an increase in Ang-2 concentration, causing increased myocardial apoptosis, increased infarction size, and impaired myocardial angiogenesis [20].

Li et al. [21] found that serum Ang-2 concentration is associated with angiopathy in T2DM, where it was significantly higher in patients with diabetic macroand microvascular complications compared to patients without angiopathy. In this study, which included 32 patients with macroangiopathy (cerebrovascular disease, heart disease, and peripheral arterial disease), and 52 patients with microangiopathy (diabetic nephropathy, diabetic retinopathy, and diabetic peripheral neuropathy) serum Ang-2 concentration was positively correlated with $\mathrm{HbA}_{1 c}$ and HOMA-IR. It may implicate that Ang-2 is closely associated with insulin resistance, glucose metabolism disorders, and vascular complications in patients with T2DM. In the same study, it was also found that Ang-2 concentration was the highest among patients with macroangiopathy and the lowest in the group of T2DM patients without any vascular complications. The in-between values of Ang-2 concentration were found in the group of T2DM patients with microangiopathy. Besides, these authors suggest that hypothetically Ang-2 has exerted pro-angiogenic activity, and high Ang-2 blood concentrations may cause local inflammation, and in the consequence the vessels' permeability may be increased [21].

Further, inflammation stimulates Ang-2 release and its binding to the Tie-2 receptor, therefore, promoting proinflammatory and prothrombotic pathways. The same authors performed a subsequent study involving a higher number of patients (240 participants vs. 120 patients in the previous one) with diabetes and confirmed that serum Ang-2 concentration is positively correlated with the number of micro and macrovascular complications of T2DM [22].

\section{Ang-2 and microangiopathy Retinopathy}

Diabetic retinopathy (DR) and diabetic macular edema (DME) have been, for years, thought to be the leading cause of blindness in the populations from the majority of developed countries [23]. Hyperglycemia leads to Ang-2 transcription, and this may lead to migration and apoptosis of retinal pericytes through Tie-2 activation. While searching for the probable explanation of this fact, one can use an animal model in which tested animals can be knocked out of specific genes. In order to explain this phenomenon, the Ang-2-deficient mice model was created, and retinal pericytes' migration, and apoptosis were observed. This proves that the inhibition of Ang-2/Tie-2 could be a potential therapeutic intervention $[24,25]$.

One of the features of DR, which may cause DME and vision impairments is vascular leakage [26]. It has been previously reported that serum Ang-2 concentration is significantly higher among T2DM patients with $\mathrm{DR}$, both non-proliferative and proliferative ones, in comparison to patients with diabetes but without DR [26]. On the basis of animal studies with streptozocininduced DR model, it was established that an increase in Ang-2 concentration was associated with vascular leakage, which can be blocked by intravitreal administration of antibodies neutralizing Ang-2 [27].

A study by Campochiaro et al. performed in patients with diabetes and DME, has shown that the administration of a vascular endothelial-protein tyrosine phosphatase, which promotes Tie-2 receptor activation (AKB-9778) for four weeks, caused DME reduction and vision improvement [28].

\section{Nephropathy}

Angiopoietins are important for glomerular capillaries in physiological conditions where they are responsible for blood flow regulation and permeability of the vascular wall. Imbalance of different growth factors, among others angiopoietins, promotes endothelial dysfunction and has been linked to the early pathological changes in glomerular function in diabetes, namely changes in blood flow and vascular wall's permeability [12].

Based on the 8-week observation of rats with streptozocin-induced diabetes, the authors concluded 
that diabetes was associated with a disproportionate increase in Ang-2 comparing to Ang-1, where Ang-1 expression was reduced in the diabetic kidney after eight weeks of the experiment [29]. Similarly, only the Ang-2 mRNA level was elevated in whole glomeruli or glomerular endothelial cells isolated from diabetic mice when compared to non-diabetic counterparts, and no changes in relation to Ang-1 were observed [30].

Additionally, it was proven that high blood glucose concentration leads to the downregulation of Ang-1 mRNA in high-glucose-treated podocytes in comparison to normal-glucose treated cells [31]. This information supports the hypothesis that Ang-2/Ang-1 may take part in diabetic glomerular disease onset and progression [32].

Also, studies performed in humans report the negative role of Ang-2 on glomerulus where Ang-2 mRNA expression was increased in glomeruli isolated from patients with diabetes comparing to specimens obtained from non-diabetic live donors and no difference was observed in Ang-1 expression [31]. Moreover, urinary Ang-2 concentration was increased in patients with T2DM and associated with albuminuria [33]. Besides, there is preliminary evidence that Ang-2 might be an independent predictor of adverse outcomes related to kidneys' function in chronic kidney disease (CKD) in patients from general as well as diabetic population [34].

Especially relevant may be the information that elevated serum Ang-2 concentration is linked to systemic inflammation in patients with CKD and may predict mortality [35]. Additionally, plasma Ang-2 concentration has also been associated with arterial stiffness, which is known to be a risk predictor of cardiovascular mortality in T2DM [36].

Most recently, it has been proven that high serum Ang-2 concentration is independently associated with the increased risk of composite outcomes of either major adverse cardiac events (MACEs) or all-cause mortality in patients with diabetic nephropathy. The authors of this work suggest that serum Ang-2 could be a potential predictive factor for MACEs in patients with diabetic nephropathy at high risk of macrovascular complications [37].

\section{Conclusion}

Patients with diabetes are at risk of microvascular and macrovascular complications [38]. Ang-2 is essential for endothelial physiology and plays an important role in vascular-related diseases as it regulates endothelial permeability and angiogenesis. A selective increase in circulating Ang-2 concentration may favor abnormal neovascularization and endothelial disruption, which are linked to both microvascular and atherosclerotic vascular complications in T2DM [17, 39]. Ang-2 plays a role in vascular diseases, and perhaps targeting
Ang/Tie signaling pathway may become a therapeutic approach because anti-angiogenic therapies are considered to be effective treatment methods in this field, especially in relation to microvascular complications of diabetes.

\section{Conflict of interest}

All the authors declare no conflict of interest in the field covered by this paper.

\section{REFERENCES}

1. Cho NH, Shaw JE, Karuranga S, et al. IDF Diabetes Atlas: Global estimates of diabetes prevalence for 2017 and projections for 2045. Diabetes Res Clin Pract. 2018; 138: 271-281, doi: 10.1016/j. diabres.2018.02.023, indexed in Pubmed: 29496507.

2. International Diabetes Federation. Diabetes and cardiovascular disease. Brussels: International Diabetes Federation 2016: 1-144.

3. Einarson TR, Acs A, Ludwig C, et al. Prevalence of cardiovascular disease in type 2 diabetes: a systematic literature review of scientific evidence from across the world in 2007-2017. Cardiovasc Diabetol. 2018; 17(1): 83, doi: 10.1186/s12933-018-0728-6, indexed in Pubmed: 29884191.

4. Microvascular Complications and Foot Care: Standards of Medical Care in Diabetes - 2019. Diabetes Care. 2019; 42(Suppl 1): S124-S138.

5. Cheloni R, Gandolfi SA, Signorelli C, et al. Global prevalence of diabetic retinopathy: protocol for a systematic review and meta-analysis. BMJ Open. 2019; 9(3): e022188, doi: 10.1136/ bmjopen-2018-022188, indexed in Pubmed: 30833309.

6. Schalkwijk CG, Ter Wee PM, Stehouwer CDA, et al. Vascular complications in diabetes mellitus: the role of endothelial dysfunction. Clin Sci (Lond). 2005; 109(2): 143-159, doi: 10.1042/CS20050025, indexed in Pubmed: 16033329.

7. Tsigkos S, Koutsilieris M, Papapetropoulos A. Angiopoietins in angiogenesis and beyond. Expert Opin Investig Drugs. 2003; 12(6): 933-941, doi: 10.1517/13543784.12.6.933, indexed in Pubmed: 12783598.

8. Papapetropoulos A, García-Cardeña G, Dengler TJ, et al. Direct actions of angiopoietin-1 on human endothelium: evidence for network stabilization, cell survival, and interaction with other angiogenic growth factors. Lab Invest. 1999; 79(2): 213-223, indexed in Pubmed: 10068209.

9. Maisonpierre PC, Suri C, Jones PF, et al. Angiopoietin-2, a natural antagonist for Tie2 that disrupts in vivo angiogenesis. Science. 1997; 277(5322): 55-60, doi: 10.1126/science.277.5322.55, indexed in Pubmed: 9204896.

10. Davis $S$, Aldrich TH, Jones $P F$, et al. Isolation of angiopoietin-1, a ligand for the TIE2 receptor, by secretion-trap expression cloning. Cell. 1996; 87(7): 1161-1169, doi: 10.1016/s00928674(00)81812-7, indexed in Pubmed: 8980223.

11. Fiedler U, Augustin HG. Angiopoietins: a link between angiogenesis and inflammation. Trends Immunol. 2006; 27(12): 552-558, doi: 10.1016/j.it.2006.10.004, indexed in Pubmed: 17045842.

12. Gnudi L. Angiopoietins and diabetic nephropathy. Diabetologia. 2016; 59(8): 1616-1620, doi: 10.1007/s00125-016-3995-3, indexed in Pubmed: 27207083.

13. Huang YQ, Li JJ, Hu L, et al. Thrombin induces increased expression and secretion of angiopoietin-2 from human umbilical vein endothelial cells. Blood. 2002; 99(5): 1646-1650, doi: 10.1182/ blood.v99.5.1646, indexed in Pubmed: 11861279.

14. Lee KW, Lip GYH, Blann AD. Plasma angiopoietin-1, angiopoietin-2, angiopoietin receptor tie-2, and vascular endothelial growth factor levels in acute coronary syndromes. Circulation. 2004; 110(16): 2355-2360, doi: 10.1161/01.CIR.0000138112.90641.7F, indexed in Pubmed: 15302795. 
15. Lorbeer R, Baumeister SE, Dörr M, et al. Circulating angiopoietin-2, its soluble receptor Tie-2, and mortality in the general population. Eur J Heart Fail. 2013; 15(12): 1327-1334, doi: 10.1093/eurjhf/ hft117, indexed in Pubmed: 23901057.

16. Tremolada G, Lattanzio R, Mazzolari G, et al. The therapeutic potential of VEGF inhibition in diabetic microvascular complications. Am J Cardiovasc Drugs. 2007; 7(6): 393-398, doi: 10.2165/00129784-200707060-00002, indexed in Pubmed: 18076206.

17. Stehouwer CD, Lambert J, Donker A, et al. Endothelial dysfunction and pathogenesis of diabetic angiopathy. Cardiovasc Res. 1997: 34(1): 55-68, doi: 10.1016/s0008-6363(96)00272-6, indexed in Pubmed: 9217873.

18. Lim HS, Lip GYH, Blann AD. Angiopoietin-1 and angiopoietin-2 in diabetes mellitus: relationship to VEGF, glycaemic control, endothelial damage/dysfunction and atherosclerosis. Atherosclerosis. 2005; 180(1): 113-118, doi: 10.1016/j.atherosclerosis.2004.11.004, indexed in Pubmed: 15823283.

19. Rasul S, Reiter MH, Ilhan A, et al. Circulating angiopoietin-2 and soluble Tie-2 in type 2 diabetes mellitus: a cross-sectional study. Cardiovasc Diabetol. 2011; 10: 55, doi: 10.1186/1475-2840-10-55, indexed in Pubmed: 21699724.

20. Tuo $Q H$, Zeng $H$, Stinnett $A$, et al. Critical role of angiopoietins/ Tie-2 in hyperglycemic exacerbation of myocardial infarction and impaired angiogenesis. Am J Physiol Heart Circ Physiol. 2008; 294(6): H2547-H2557, doi: 10.1152/ajpheart.01250.2007, indexed in Pubmed: 18408125.

21. Li Li, Qian L, Yu ZQ. Serum angiopoietin-2 is associated with angiopathy in type 2 diabetes mellitus. J Diabetes Complications. 2015; 29(4): 568-571, doi: 10.1016/j.jdiacomp.2015.02.006, indexed in Pubmed: 25754501.

22. Li Li, ZHeng-Qing $\mathrm{Yu}$, Juan-Yu Hu, et al. Association between interleukin-19 and angiopoietin-2 with vascular complications in type 2 diabetes. J Diabetes Investig. 2016; 7(6): 895-900, doi: 10.1111/jdi.12519, indexed in Pubmed: 27182008.

23. Ciulla TA, Amador AG, Zinman B. Diabetic retinopathy and diabetic macular edema: pathophysiology, screening, and novel therapies. Diabetes Care. 2003; 26(9): 2653-2664, doi: 10.2337/ diacare.26.9.2653, indexed in Pubmed: 12941734.

24. Cai J, Kehoe O, Smith GM, et al. The angiopoietin/Tie-2 system regulates pericyte survival and recruitment in diabetic retinopathy. Invest Ophthalmol Vis Sci. 2008; 49(5): 2163-2171, doi: 10.1167/ iovs.07-1206, indexed in Pubmed: 18436850.

25. Pfister F, Feng $Y$, vom Hagen $F$, et al. Pericyte migration: a novel mechanism of pericyte loss in experimental diabetic retinopathy. Diabetes. 2008; 57(9): 2495-2502, doi: 10.2337/db08-0325, indexed in Pubmed: 18559662.

26. Khalaf $\mathrm{N}$, Helmy $\mathrm{H}$, Labib $\mathrm{H}$, et al. Role of angiopoietins and Tie-2 in diabetic retinopathy. Electron Physician. 2017; 9(8): 5031-5035, doi: 10.19082/5031, indexed in Pubmed: 28979738.

27. Yun JH, Park SW, Kim JH, et al. Angiopoietin 2 induces astrocyte apoptosis via $\alpha \mathrm{v} \beta 5$-integrin signaling in diabetic retinopathy. Cell
Death Dis. 2016: 7: e2101, doi: 10.1038/cddis.2015.347, indexed in Pubmed: 26890140.

28. Campochiaro PA, Sophie R, Tolentino M, et al. Treatment of diabetic macular edema with an inhibitor of vascular endothelialprotein tyrosine phosphatase that activates Tie2. Ophthalmology. 2015; 122(3): 545-554, doi: 10.1016/j.ophtha.2014.09.023, indexed in Pubmed: 25439435.

29. Rizkalla B, Forbes JM, Cao Z, et al. Temporal renal expression of angiogenic growth factors and their receptors in experimental diabetes: role of the renin-angiotensin system. J Hypertens. 2005; 23(1): 153-164, doi: 10.1097/00004872-200501000-00026, indexed in Pubmed: 15643138.

30. Jeansson M, Gawlik A, Anderson G, et al. Angiopoietin-1 is essential in mouse vasculature during development and in response to injury. J Clin Invest. 2011; 121(6): 2278-2289, doi: 10.1172/ $\mathrm{JCl} 46322$, indexed in Pubmed: 21606590.

31. Dessapt-Baradez C, Woolf AS, White KE, et al. Targeted glomerular angiopoietin-1 therapy for early diabetic kidney disease. J Am Soc Nephrol. 2014; 25(1): 33-42, doi: 10.1681/ASN.2012121218, indexed in Pubmed: 24009238.

32. Gnudi L. Angiopoietins and diabetic nephropathy. Diabetologia. 2016; 59(8): 1616-1620, doi: 10.1007/s00125-016-3995-3, indexed in Pubmed: 27207083.

33. Chen S, Li H, Zhang C, et al. Urinary angiopoietin-2 is associated with albuminuria in patients with type 2 diabetes mellitus. Int J Endocrinol. 2015; 2015: 163120, doi: 10.1155/2015/163120, indexed in Pubmed: 25873946.

34. Tsai YC, Chiu YW, Tsai JC, et al. Association of angiopoietin-2 with renal outcome in chronic kidney disease. PLoS One. 2014; 9(10): e108862, doi: 10.1371/journal.pone.0108862, indexed in Pubmed: 25279852.

35. David S, John SG, Jefferies HJ, et al. Angiopoietin-2 levels predict mortality in CKD patients. Nephrol Dial Transplant. 2012; 27(5): 1867-1872, doi: 10.1093/ndt/gfr551, indexed in Pubmed: 21976741.

36. Chang FC, Chiang WC, Tsai MH, et al. Angiopoietin-2-induced arterial stiffness in CKD. J Am Soc Nephrol. 2014; 25(6): 1198-1209, doi: 10.1681/ASN.2013050542, indexed in Pubmed: 24511140.

37. Tsai YC, Lee CS, Chiu YW, et al. Angiopoietin-2, renal deterioration, major adverse cardiovascular events and all-cause mortality in patients with diabetic nephropathy. Kidney Blood Press Res. 2018; 43(2): 545-554, doi: 10.1159/000488826, indexed in Pubmed: 29642068.

38. Chawla A, Chawla R, Jaggi S. Microvasular and macrovascular complications in diabetes mellitus: Distinct or continuum? Indian J Endocrinol Metab. 2016; 20(4): 546-551, doi: 10.4103/22308210.183480, indexed in Pubmed: 27366724.

39. Kim M, Allen B, Korhonen EA, et al. Opposing actions of angiopoietin-2 on Tie2 signaling and FOXO1 activation. J Clin Invest. 2016; 126(9): 3511-3525, doi: 10.1172/JCl84871, indexed in Pubmed: 27548529. 